\title{
Surveillance de la coqueluche au Canada : tendances jusqu'à
} 2012

\author{
Smith $\mathrm{T}^{1 *}$, Rotondo $\mathrm{J}^{1}$, Desai $\mathrm{S}^{1}$ et Deehan $\mathrm{H}^{1}$.
}

1 Agence de la santé publique du Canada, Centre de l'immunisation et des maladies respiratoires infectieuses, Ottawa (ON)

* Auteure-ressource : tiffany.smith@phac-aspc.gc.ca

\section{Résumé}

Objectif : Le présent rapport a pour objectif de présenter sommairement l'activité de la coqueluche au Canada.

Méthodes: Une analyse descriptive de l'incidence de la coqueluche selon l'année, le groupe d'âge, le genre et la province ou le territoire a été effectuée avec des données de surveillance nationales, des données cliniques administratives et des données statistiques de l'état civil.

Résultats : La coqueluche est une maladie endémique cyclique au Canada qui connaît des pics d'activité tous les deux à cinq ans. Le Canada a connu un déclin de l'activité de la coqueluche après avoir adopté des programmes d'immunisation de routine contre la coqueluche. C'est chez les nourrissons et les enfants que l'incidence de la coqueluche est la plus élevée. Les hospitalisations et les décès sont plus fréquents chez les nourrissons, particulièrement chez ceux de moins de trois mois. Les tendances en ce qui concerne la coqueluche varient selon la province et le territoire. Le Canada a connu une augmentation notable de l'incidence de la maladie en 2012. Les raisons de cette augmentation sont inconnues.

Conclusion : Nous pourrions mieux comprendre l'épidémiologie de la coqueluche au Canada en améliorant les méthodes de surveillance de la maladie. Bien que le pic d'activité observée en 2012 soit peut-être un événement isolé, il faudrait envisager d'entreprendre des travaux plus poussés pour aider les provinces et les territoires à répondre aux éclosions de la maladie, et trouver notamment des ressources et des outils de recherche rapides.

\section{Introduction}

Au Canada, la coqueluche fait l'objet d'une surveillance nationale depuis 1924 et est l'une des maladies ciblées par les programmes d'immunisation des enfants depuis 1943. II existe des programmes d'immunisation systématique des nourrissons, des enfants, des adolescents et des adultes d'un bout à l'autre du Canada, mais les vaccins utilisés et le calendrier de vaccination est variable d'une province ou d'un territoire à l'autre ${ }^{1}$. Bien que la mise en place de programmes d'immunisation des enfants ait permis de réduire considérablement l'incidence de la maladie, la coqueluche demeure l'une des maladies évitables par la vaccination la plus souvent signalée au Canada. Au début de l'année 2012, l'Agence de la santé publique du Canada (I'Agence) a reçu des rapports d'une augmentation de l'activité de la coqueluche dans plusieurs provinces et territoires, ce qui l'a incitée à revoir les données sur la coqueluche. Le but du présent rapport est de présenter sommairement l'activité de la coqueluche au Canada depuis que cette maladie est devenue une maladie à déclaration obligatoire, en mettant l'accent sur l'activité récente. 


\section{Méthodes}

\section{SOURCES DES DONNÉES}

\section{Rapports de cas à l'échelle nationale}

Les cas confirmés de coqueluche signalés à l'échelle nationale de 1924 à 2011 ont été extraits de la base de données du Système canadien de surveillance des maladies à déclaration obligatoire en mars $2013^{2}$. Des définitions de cas nationales pour la coqueluche ont été publiées en $1991^{3}, 2000^{4}$ et $2009^{5}$. Avant l'an 2000 , on parlait de cas confirmés lorsqu'on pouvait isoler la bactérie Bordetella pertussis et qu'il y avait présence de symptômes cliniquement compatibles. De 2000 à 2008, la définition de cas exigeait une confirmation en laboratoire (par la culture d'un spécimen clinique approprié ou la détection de l'ADN) ou l'établissement d'un lien épidémiologique avec un cas confirmé en laboratoire et un des nombreux symptômes cliniques. En 2009, la définition de cas a été précisée davantage afin d'exiger que les cas où l'ADN de B. pertussis était détecté présentent également des symptômes cliniquement compatibles. Les cas confirmés de coqueluche pour 2012 ont été obtenus directement des provinces et des territoires en mars 2013 par le Centre de l'immunisation et des maladies respiratoires infectieuses de l'Agence et sont des données préliminaires.

\section{Hospitalisations}

Les cas de coqueluche signalés dans le cadre du Programme canadien de surveillance active de l'immunisation (IMPACT) de 1991 à 2012 ont été extraits de la base de données du programme en avril $2013^{6}$. Les données de 2012 sont des données préliminaires. Depuis 1991, le programme IMPACT fournit à l'Agence des données plus enrichies sur les cas de coqueluche chez les enfants traités dans des centres de soins tertiaires au Canada. Initialement, ces données provenaient de 5 centres, puis de 10 en 1993, de 11 en 1995 et de 12 en 1999.

Les données sur les hospitalisations en soins de courte durée liées à la coqueluche recueillies de 1995 à 2010 ont été extraites de la base de données sur la morbidité hospitalière de l'Institut canadien d'information sur la santé en juin 2013. Ces dates ont été choisies, car les ensembles de données étaient complets pour chacune de ces années. Les hospitalisations dues à la coqueluche ont été définies comme celles dont le diagnostic au moment du congé (tous les niveaux de diagnostic), de catégorie 9 ou 10 dans la classification internationale des maladies, correspondait à un diagnostic de coqueluche (0330, 0339, 4843, A370 ou A379). Les critères d'exclusion comprenaient les transferts entre hôpitaux et les réadmissions, celles-ci étant définies comme plus d'une hospitalisation pour le même cas en moins d'un an.

\section{Statistiques de l'état civil}

Des données sur la mortalité ont été obtenues de la Base de données sur les décès, qui est une base de données nationale renfermant des renseignements démographiques et médicaux (cause initiale de décès) recueillis chaque année dans les registres de statistiques de l'état civil sur les décès de l'ensemble des provinces et des territoires du Canada ${ }^{7}$. Les décès dont la cause initiale était la coqueluche ont été décelés au moyen des mêmes codes de la classification internationale des maladies énumérés précédemment.

\section{Analyse}

Une analyse descriptive de l'incidence de la coqueluche selon l'année, le groupe d'âge, le genre et la province ou le territoire a été effectuée. Les données sur la population qui ont servi à calculer les taux d'incidence ont été obtenues auprès de Statistique Canada ${ }^{8}$. Comme les données envoyées au Système canadien de surveillance des maladies à déclaration obligatoire sont regroupées par groupe d'âge, les taux selon l'âge ont été calculés seulement pour les groupes d'âge suivants : moins de 1 an, 1 à 4 ans, 5 à 9 ans, 10 à 14 ans, 15 à 19 ans, 20 à 24 ans, 25 à 29 ans, 30 à 39 ans, 40 à 59 ans, et 60 ans et plus. Les taux d'incidence de cas confirmés sont des taux pour 100000 habitants. Les provinces et les territoires qui n'ont pas été en mesure de fournir leur compte de cas respectif n'ont pas été inclus dans le dénominateur pour l'année correspondante. La variation de pourcentage annuelle a été utilisée pour décrire la variation des taux de déclaration au fil du temps. Des ratios du taux d'incidence ont été calculés pour examiner les différences entre les sexes. 


\title{
Résultats
}

\section{Incidence annuelle}

Au cours des 5 années qui ont précédé la mise en place du programme de vaccination, le taux d'incidence moyen de la coqueluche était de 156 cas pour 100000 habitants. Après l'introduction du vaccin, le taux d'incidence moyen annuel a chuté à aussi peu que 7 cas pour 100000 habitants de 1984 à 1988 (figure 1). Une résurgence de la coqueluche a été observée en 1989-1990 et le taux d'incidence a atteint un sommet de 34,9 cas pour 100 000 habitants en 1994. Après 1998, le taux d'incidence est tombé à 2 cas pour 100000 habitants en 2011, soit le taux le plus faible jamais enregistré au Canada. Une augmentation du taux d'incidence national multipliée par 7 (13,9 pour 100000 habitants) a été observée en 2012. Les tendances au fil du temps révèlent la présence de pics d'activité tous les 2 à 5 ans. Ces pics sont maintenant moins évidents, car l'incidence de la maladie a diminué.

Figure 1: Taux d'incidence et cas déclarés (pour 100000 habitants) de coqueluche au Canada, par année, de 1924 à $2012^{*}$

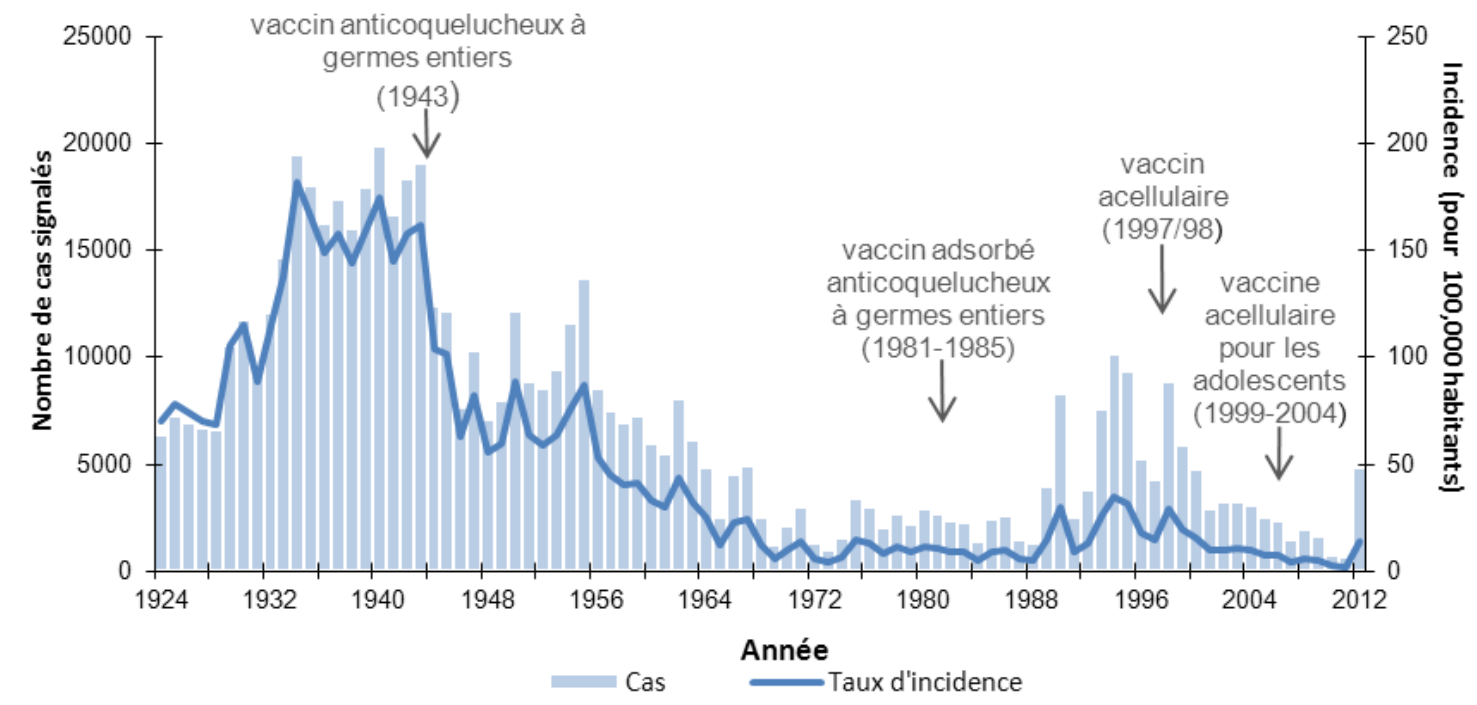

\begin{abstract}
*Les données de cas de 1924 à 2011 proviennent du Système canadien de surveillance des maladies à déclaration obligatoire. Les données de cas pour 2012 ont été obtenues directement des provinces et des territoires par le Centre de l'immunisation et des maladies respiratoires infectieuses, et sont des données préliminaires. L'Île-du-Prince-Édouard n'a pas déclaré les cas de la province de 1924 à 1928, Terre-Neuve-et-Labrador n'a pas déclaré ses cas jusqu'en 1949, le Yukon n'a pas déclaré les cas du territoire de 1924 à 1955, les Territoires du Nord-Ouest n'ont pas déclaré leurs cas de 1924 à 1958, les données du Nunavut pour 1999 sont des données partielles, le Nunavut n'a aucune donnée pour 2007 et 2009, et ses données pour 2008, 2010 et 2011 sont des données préliminaires. Les données démographiques (estimations annuelles au $1^{\text {er }}$ juillet) ont été obtenues auprès de Statistique Canada.
\end{abstract}

En général, la tendance dans les données sur les hospitalisations attribuables à la coqueluche était semblable à la tendance observée dans les rapports de maladies à déclaration obligatoire (figure 2). Les rapports de cas à l'échelle nationale et les hospitalisations en soins de courte durée ont diminué de $92 \%$ de 1995 à 2010; les rapports de cas ont chuté, passant de 9308 (31,8 pour 100000 habitants) en 1995 à 748 (2,2 pour 100 000 habitants) en 2010 , et, selon la base de données sur la morbidité hospitalière, les hospitalisations ont elles aussi diminué, passant de 2016 (6,8 pour 100000 habitants) en 1995 à 168 (0,5 pour 100000 habitants) en 2010. Durant cette même période, les hospitalisations enregistrées dans le cadre du programme IMPACT ont diminué de $82 \%$, passant de 264 en 1995 à 48 en 2010. Toutes les sources de données ont fait état de pics d'activité en 1998 ainsi que de légères augmentations en 2002 et 2008. De légères augmentations des hospitalisations ont aussi été observées en 2004 (base de données sur la morbidité hospitalière : $16 \%$; programme IMPACT : $65 \%$ ) et 2009 (base de données sur la morbidité hospitalière : $20 \%$; programme IMPACT : $26 \%$ ), tandis que ces années-là, les rapports de cas ont décliné par rapport à l'année précédente de $4 \%$ et de $18 \%$, respectivement. De façon semblable aux rapports de cas à l'échelle nationale, les cas déclarés 
dans les hôpitaux participant au programme IMPACT ont augmenté en 2012, mais pas aussi radicalement que le taux d'incidence national de la maladie (augmentation multipliée par 2).

Figure 2 : Nombre de cas déclarés et hospitalisations dues à la coqueluche au Canada, par année, de 1995 à $2012^{*}$

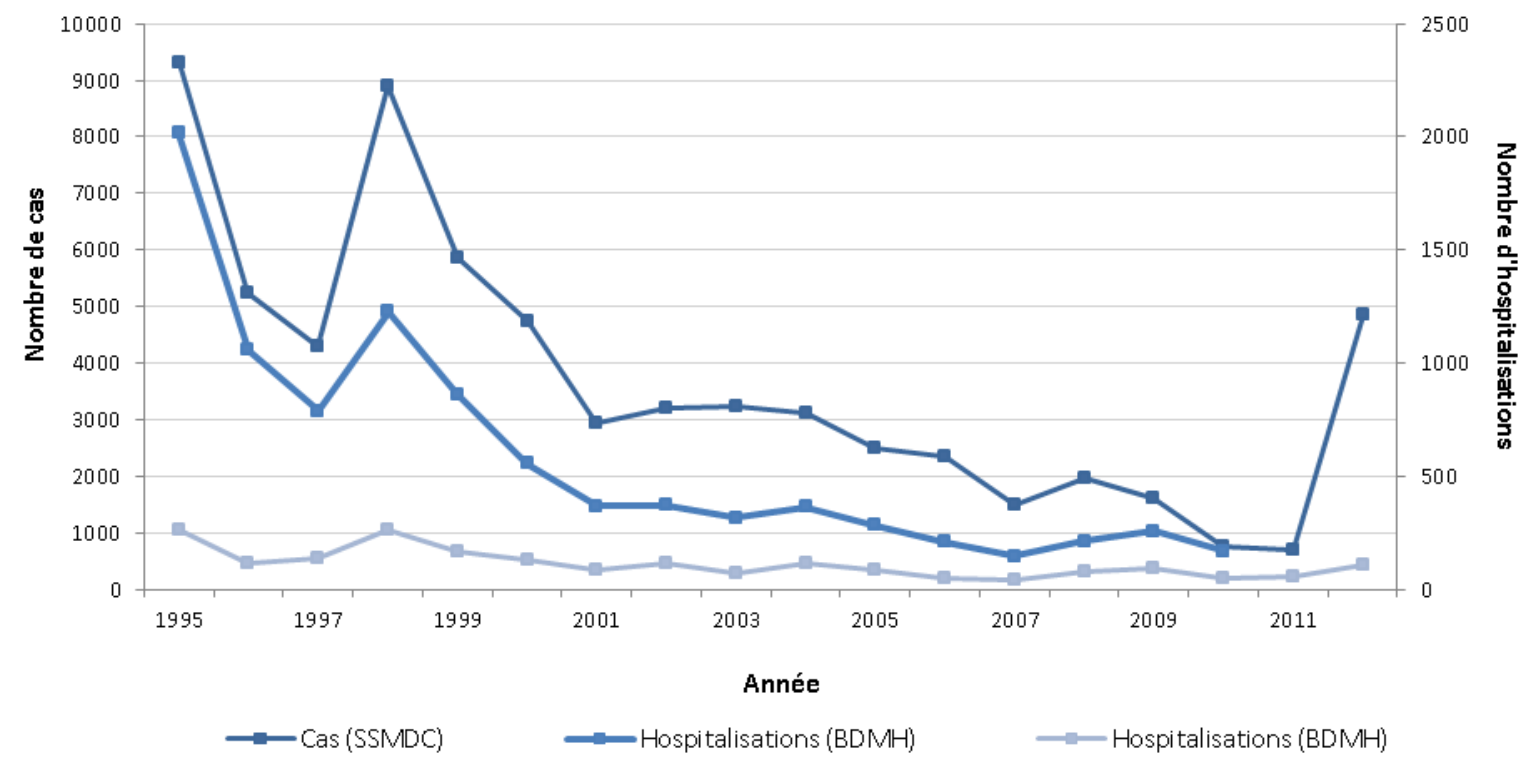

*Les données de cas de 1995 à 2011 proviennent du Système canadien de surveillance des maladies à déclaration obligatoire. Les données de cas pour 2012 ont été obtenues directement des provinces et des territoires par le Centre de l'immunisation et des maladies respiratoires infectieuses, et sont des données préliminaires. Les données du Nunavut pour 1999 sont des données partielles, le Nunavut n'a aucune donnée pour 2007 et 2009, et ses données pour 2008, 2010 et 2011 sont des données préliminaires; les données recueillies dans le cadre du programme IMPACT pour 2012 sont des données préliminaires.

\section{Répartition selon l'âge}

L'incidence de la coqueluche est plus élevée chez les nourrissons et les enfants, et elle diminue brusquement chez les personnes de plus de 14 ans (figure 3). Les taux d'incidence moyens les plus élevés observés de 2005 à 2011 étaient de 72,2 cas pour 100000 habitants chez les nourrissons de moins d'un an (261 cas par année en moyenne), de 25,6 cas pour 100000 habitants chez les enfants de 1 à 4 ans (362 cas par année en moyenne) et de 16,0 cas pour 100000 habitants chez les enfants de 10 à 14 ans (328 cas par année en moyenne). En comparaison, l'incidence moyenne de la coqueluche chez les personnes de 15 ans et plus était de 1,6 cas pour 100000 habitants de 2005 à 2011 (428 cas par année en moyenne).

De 2005 à 2011, l'incidence de la coqueluche a diminué dans tous les groupes d'âge, en particulier chez les jeunes de 10 à 14 ans (diminution de 84 \%) et chez les 15 à 19 ans (diminution de $81 \%$ ). En 2012, une augmentation de l'incidence de la maladie a été observée au pays dans tous les groupes d'âge, mais les taux d'incidence les plus élevés ont été observés chez les moins d'un an $(120,8$ pour 100000 habitants; $n=460)$ et chez les jeunes de 10 à 14 ans (64,1 pour 100000 habitants; $n=1203)$. Les taux d'incidence les plus élevés dans la majorité des provinces et des territoires ont été observés chez les enfants de moins de 15 ans. 
Figure 3 : Taux d'incidence (pour 100000 habitants) des cas déclarés de coqueluche au Canada, selon le groupe d'âge (en années) et selon l'année, de 1980 à 2012*

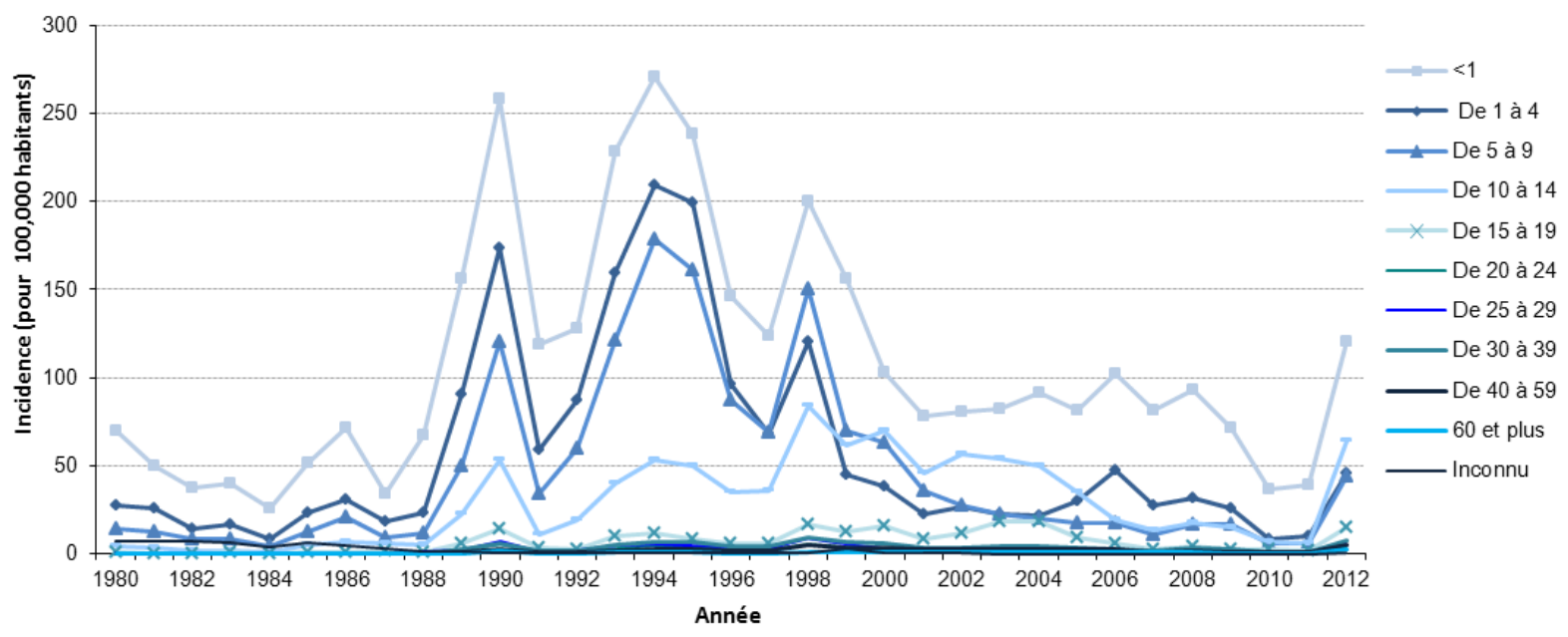

*Les données de cas de 1980 à 2011 proviennent du Système canadien de surveillance des maladies à déclaration obligatoire. Les données de cas pour 2012 ont été obtenues directement des provinces et des territoires par le Centre de l'immunisation et des maladies respiratoires infectieuses, et sont des données préliminaires. Les données du Nunavut pour 1999 sont des données partielles, le Nunavut n'a aucune donnée pour 2007 et 2009, et ses données pour 2008, 2010 et 2011 sont des données préliminaires. Les données démographiques (estimations annuelles au $1^{\mathrm{er}}$ juillet) ont été obtenues auprès de Statistique Canada.

Des fluctuations dans la proportion des cas de coqueluche selon le groupe d'âge ont été observées au cours des 20 dernières années, particulièrement chez les enfants âgés de 1 à 9 ans et chez les adolescents âgés de 10 à 19 ans (figure 4). Dans les années 1990, la proportion la plus élevée de cas a été observée dans les groupes d'âge des 1 à 9 ans (62\% par année en moyenne). De 2000 à 2005, la proportion de cas chez les adolescents (c.-à-d. les 10 à 19 ans) a augmenté et était en moyenne de $42 \%$ par année, tandis que la proportion de cas chez les 1 à 9 ans est tombée à $30 \%$ par année en moyenne. De 2006 à 2011, les cas chez les 10 à 19 ans ont chuté à $23 \%$ par année en moyenne, tandis que le nombre de cas chez les 1 à 9 ans a augmenté très légèrement pour s'inscrire à $38 \%$ par année en moyenne. Alors que ces grandes fluctuations se produisaient, de légères augmentations étaient observées chez les nourrissons (< 1 an) et chez les adultes (20 ans et plus); en effet, les moyennes annuelles respectives de $12 \%$ et $10 \%$ de cas dans les années 1990 sont passées à $18 \%$ et $21 \%$ entre 2006 et 2011. En 2012, $10 \%$ des cas étaient âgés de moins d'un an, $32 \%$ étaient âgés de 1 à 9 ans, $31 \%$ étaient âgés de 10 à 19 ans et $27 \%$ avaient 20 ans et plus.

Par contre, la distribution selon l'âge des cas hospitalisés en soins de courte durée n'a pas fluctué considérablement au fil du temps et la proportion des hospitalisations est toujours plus élevée chez les enfants de moins d'un an. De 1995 à 2010, chaque année, en moyenne, 69 \% des admissions liées à la coqueluche étaient des enfants de moins d'un an (intervalle de 59 à $78 \%$ ). Dans ce groupe d'âge, la majorité des admissions était de jeunes nourrissons, la proportion moyenne annuelle des cas âgés de moins de 3 mois étant de $62 \%$ (intervalle de 49 à $71 \%)$. 
Figure 4 : Répartition selon l'âge des cas de coqueluche au Canada, de 1990 à $2012^{*}$

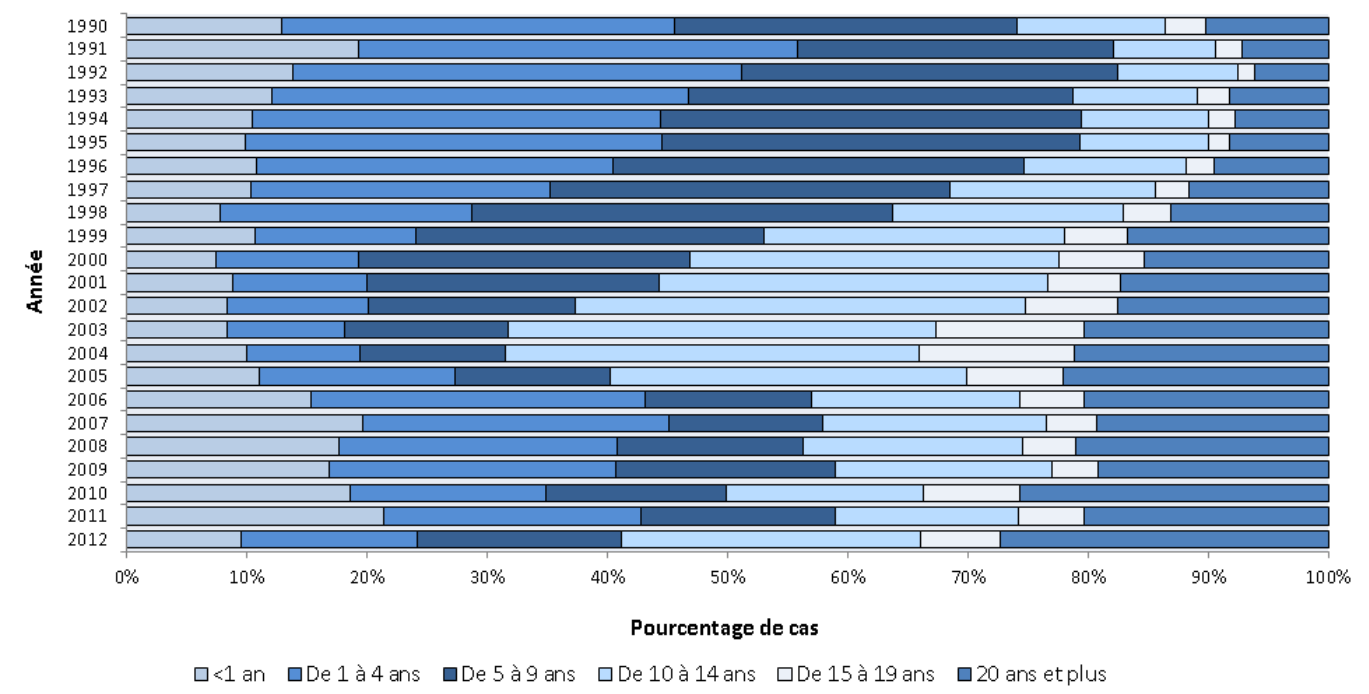

*Les données de cas de 1990 à 2011 proviennent du Système canadien de surveillance des maladies à déclaration obligatoire. Les données de cas pour 2012 ont été obtenues directement des provinces et des territoires par le Centre de l'immunisation et des maladies respiratoires infectieuses, et sont des données préliminaires. Les données du Nunavut pour 1999 sont des données partielles, le Nunavut n'a aucune donnée pour 2007 et 2009, et ses données pour 2008, 2010 et 2011 sont des données préliminaires.

\section{Répartition selon le sexe}

De 1990 à 2012, le nombre de cas de coqueluche déclarés au pays et le taux d'incidence ont été systématiquement plus élevés chez les femmes, dans tous les groupes d'âge. Pendant cette période, le rapport médian annuel hommes-femmes du taux d'incidence était de 0,87:1 (variation de 0,81:1 à 0,96:1). Chaque année, en moyenne, les femmes représentaient $54,0 \%$ des cas, les hommes $45,8 \%$ des cas et, 0,2 \% des cas étaient de sexe inconnu. De la même façon, de 1995 à 2010, le rapport médian hommes-femmes du taux d'incidence d'hospitalisations était de 0,89:1 (variation de 0,73:1 à 1,05:1) et, en moyenne, chaque année, les femmes représentaient $53,5 \%$ des cas.

\section{Répartition géographique}

On s'attend à une asynchronie du moment et du lieu géographique des pics cycliques de coqueluche échelonnées sur 2 à 5 ans. Bien que le Canada ait connu un déclin global de l'incidence de la coqueluche de la fin des années 1990 à 2011, le moment, l'ampleur et la tendance générale de la diminution de l'activité ont varié au sein des provinces et des territoires (figure 5). De 1995 à 2011, on a observé des pics de cas de coqueluche confirmés en laboratoire dans toutes les provinces et territoires. Le taux d'incidence le plus élevé par province, durant cette période, a été observé en 1995 au Manitoba, en Ontario, au Nouveau-Brunswick et en NouvelleÉcosse, en 1996 en Alberta, en 1997 à l'Île-du-Prince-Édouard, en 1998 au Québec, en 1999 en Saskatchewan, à Terre-Neuve-et-Labrador, dans les Territoires du Nord-Ouest et au Nunavut, et en 2000 en ColombieBritannique et au Yukon. La différence absolue entre le taux d'incidence le plus élevé et le taux d'incidence le plus faible au sein d'une province ou d'un territoire, durant cette période, variait entre 18 cas pour 100 000 habitants et une différence beaucoup plus grande de 489 cas pour 100000 habitants, les fluctuations importantes étant plus courantes dans les provinces et les territoires moins densément peuplés. De l'année où chaque province ou territoire a enregistré un pic jusqu'à 2011, ils ont tous connu un déclin du taux d'incidence de plus de $75 \%$, la grande majorité ayant connu une baisse de plus de $90 \%$.

Les augmentations du taux d'incidence à l'échelle nationale sont habituellement le résultat de pics observées dans plusieurs provinces et territoires. L'augmentation nationale observée en 1998 était due à l'augmentation de l'activité au Manitoba, en Ontario, au Québec et au Nouveau-Brunswick. C'est également ce qui s'est passé en 2012, lorsqu'une augmentation de l'incidence par rapport à 2011 a été observée dans 9 provinces et territoires 
sur 13. À l'échelle provinciale et territoriale, ces augmentations variaient d'une augmentation négligeable de 0,7 cas pour 100000 habitants à une augmentation plus substantielle de 152 cas pour 100000 habitants. Au sein des provinces et des territoires ayant connu des augmentations substantielles, les causes étaient variables et allaient d'éclosions à la grandeur de la province, à des éclosions régionales et à des éclosions localisées. L'augmentation de l'activité la plus remarquable a été observée au Nouveau-Brunswick, en raison d'une éclosion à l'échelle provinciale représentant environ le tiers du nombre de cas au pays en 2012.

\section{Mortalité}

De 2000 à 2009, Statistique Canada a fait état de 6 décès dont la cause initiale était la coqueluche. Chaque année, de 0 à 2 décès ont été signalés et tous étaient des nourrissons de moins d'un an. Toutefois, il s'agit sans doute d'une sous-estimation du nombre total de décès attribuables à la coqueluche au Canada. D'après les données du programme IMPACT, 11 des 858 cas de coqueluche signalés de 2000 à 2009 dans le cadre de ce programme sont décédés (1,2\%). De 1991 à 2012, 30 décès ont été signalés par l'intermédiaire du programme IMPACT, ce qui représente de 0 à 4 décès par année (figure 6). La majorité des décès était des nourrissons âgés de moins de 2 mois (78\%). Tous les enfants décédés, sauf un, étaient des nourrissons n'ayant pas été vaccinés contre la coqueluche et $78 \%$ des décès étaient des enfants considérés comme étant auparavant en santé. Les causes de décès étaient notamment : détresse et insuffisance respiratoire, pneumonie, hypoxie, hypertension artérielle pulmonaire, hémorragie pulmonaire, choc septique fulminant, défaillance cardiovasculaire et arythmie aiguë.

*Les données de cas de 1995 à 2011 proviennent du Système canadien de surveillance des maladies à déclaration obligatoire. Les données de cas pour 2012 ont été obtenues directement des provinces et des territoires par le Centre de l'immunisation et des maladies respiratoires infectieuses, et sont des données préliminaires. Les données du Nunavut pour 1999 sont des données partielles, le Nunavut n'a aucune donnée pour 2007 et 2009, et ses données pour 2008, 2010 et 2011 sont des données préliminaires. Les données démographiques (estimations annuelles au $1^{\mathrm{er}}$ juillet) ont été obtenues auprès de Statistique Canada.
Figure 5 : Tendances de l'incidence annuelle de la coqueluche (pour 100000 habitants), selon la province ou le territoire, de 1995 à 2012

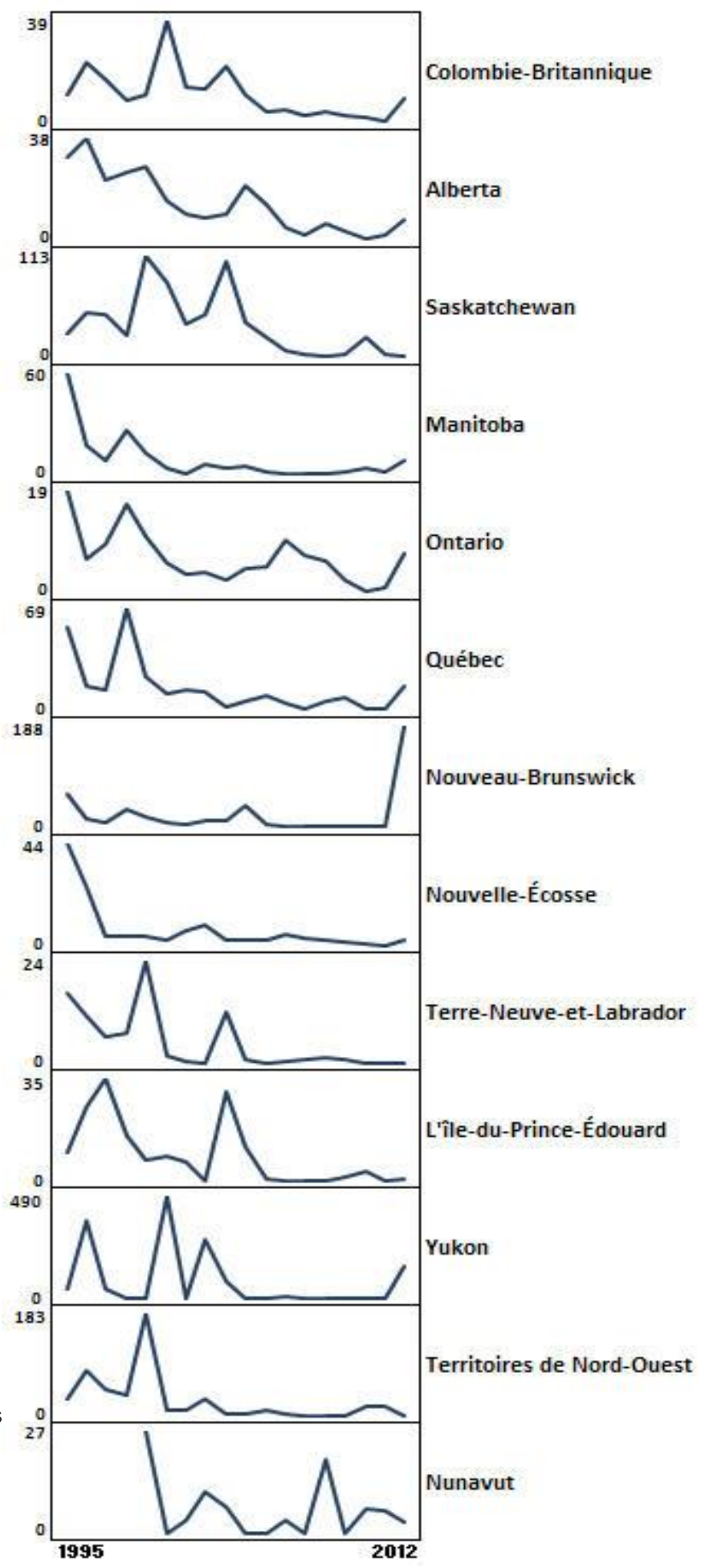


Figure 6 : Nombre de cas de coqueluche et de décès, programme IMPACT, de 1991 à 2012 *

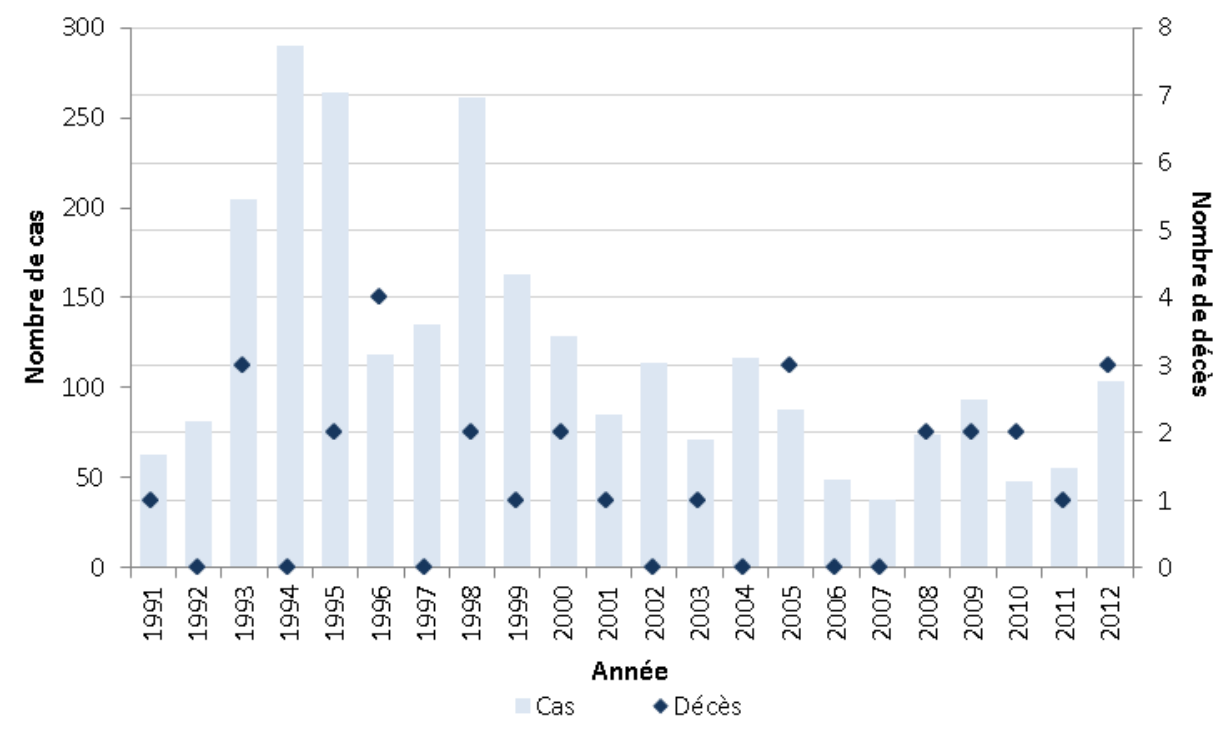

*Les données pour 2012 sont des données préliminaires.

\section{Discussion}

La coqueluche est une maladie endémique cyclique au Canada qui connaît des pics d'activité tous les deux à cinq ans. Bien que le Canada ait connu une résurgence de la maladie dans les années 1990, il a connu dans l'ensemble un déclin de l'activité après l'adoption de programmes d'immunisation de routine contre la coqueluche. Ce déclin a d'abord été observé avec l'introduction du vaccin à germes entiers en 1943, puis après l'introduction du vaccin acellulaire en 1997-1998 et de 2005 à 2011 après l'adoption de programmes d'immunisation des adolescents dans l'ensemble du pays ${ }^{9}$. Tel qu'il a été mentionné précédemment, la résurgence observée dans les années 1990 était probablement due à une combinaison de facteurs, y compris la faible efficacité du vaccin à germes entiers introduit de 1981 à 1985, de même que la sensibilisation accrue des médecins, et l'amélioration des diagnostics d'infections de coqueluche et des déclarations de la maladie ${ }^{10}$. D'un point de vue géographique, toutes les provinces et tous les territoires ont connu un déclin de l'incidence de la coqueluche au cours des sept années qui ont précédé 2012; néanmoins, les tendances de la maladie, notamment en ce qui concerne le moment et l'ampleur des pics cycliques, étaient propres à chacun.

L'incidence de la coqueluche est toujours plus élevée chez les nourrissons, les hospitalisations et les décès aussi, particulièrement chez les trois mois et moins. Ce groupe présente un intérêt particulier étant donné que les enfants sont trop jeunes pour bénéficier de la protection d'un vaccin. Différentes approches ont été essayées, y compris le « coconnage » et l'immunisation des femmes enceintes pour favoriser l'immunisation passive du nourrisson $^{11,12,13}$. Le Comité consultatif national de l'immunisation du Canada recommande de vacciner les femmes enceintes s'il y a une éclosion régionale de coqueluche ${ }^{14}$.

L'augmentation de l'incidence observée au pays en 2012 était due à des éclosions dans plusieurs provinces et territoires. Une augmentation de l'incidence a été observée dans tous les groupes d'âge, partout au pays, et les taux d'incidence les plus élevés ont été observés chez les moins d'un an. Cela dit, les groupes d'âge les plus touchés variaient d'une province ou d'un territoire à l'autre. Comme pour les rapports de cas à l'échelle nationale, les cas déclarés dans les hôpitaux participant au programme IMPACT ont augmenté en 2012, mais pas aussi radicalement, ce qui laisse entendre que l'augmentation des cas n'était pas liée à une augmentation de la gravité.

Une augmentation de l'activité de la coqueluche a également été observée récemment dans d'autres pays et plusieurs raisons sont proposées dans la littérature : baisse de l'immunité ${ }^{15}$, diminution de la stimulation de l'immunité naturelle ${ }^{16}$, taux de détection plus élevés ${ }^{17}$, modifications génétiques de la bactérie ${ }^{18}$ et regroupement 
des personnes non vaccinées ${ }^{19}$. Selon les données disponibles à l'échelle nationale, il est difficile de déterminer les facteurs ayant contribué aux augmentations observées au Canada en 2012. À l'heure actuelle, très peu de données ont été recueillies à l'échelle nationale et, souvent, il s'agit de données globales qui n'incluent pas les antécédents vaccinaux des cas. Le Laboratoire national de microbiologie n'effectue pas à l'heure actuelle une surveillance de routine des souches de coqueluche en circulation. D'après les résultats validés des enquêtes nationales sur la vaccination des enfants de 2009, la couverture vaccinale est élevée; $98 \%$ pour 4 doses et plus chez les enfants de 2 ans et $89 \%$ pour 5 doses et plus chez les enfants de 7 ans $^{20}$. Cependant, certains programmes d'immunisation et la couverture vaccinale au sein des populations et des sous-populations au Canada n'ont pas encore fait l'objet d'une évaluation systématique.

\section{Limites}

Compte tenu de la nature passive du Système canadien de surveillance des maladies à déclaration obligatoire, on s'attend à ce que les cas déclarés représentent une sous-estimation du fardeau réel de la maladie, particulièrement chez les adolescents et les adultes. L'incidence de la coqueluche chez les adultes est sans doute plus élevée que celle signalée, car les symptômes sont généralement légers, et il est rare que les adultes souffrant de toux persistante fassent l'objet d'un dépistage de $B$. pertussis. En outre, les hospitalisations liées à la coqueluche enregistrées dans la base de données sur la morbidité hospitalière sont codées en fonction du diagnostic du médecin, tel que ce diagnostic est indiqué dans le dossier médical, et ne correspondent pas nécessairement à la définition de cas nationale (c.-à-d. elles ne sont peut-être pas fondées sur des résultats de laboratoire). Par conséquent, le nombre d'hospitalisations en soins de courte durée liées à la coqueluche présenté dans ce rapport pourrait être une surestimation du fardeau réel.

\section{Conclusion}

En conclusion, nous pourrions mieux comprendre l'épidémiologie de la coqueluche au Canada en améliorant les méthodes de surveillance de la maladie. Une surveillance de la coqueluche à l'échelle nationale qui consignerait également les antécédents vaccinaux, certaines caractéristiques microbiologiques et tout signe d'éclosions pourraient éclairer les mesures de santé publique à prendre. Bien que le pic d'activité observée en 2012 soit peutêtre un événement isolé, il faudrait envisager d'entreprendre des travaux plus poussés pour aider les provinces et les territoires à répondre aux éclosions de la maladie, et trouver notamment des ressources et des outils de recherche rapides.

\section{Références}

1. Agence de la santé publique du Canada. Programmes d'immunisation subventionnés par l'État au Canada Calendrier d'immunisation systématique des nourrissons et des enfants incluant les programmes de rappel (en date de mars 2013).

http://www.phac-aspc.gc.ca/im/ptimprog-progimpt/table-1-fra.php

2. Agence de la santé publique du. Maladies à déclaration obligatoire au pays.

http://www.phac-aspc.gc.ca/aids-sida/about/dis-fra.php

3. Santé et Bien-être social Canada. Programme canadien de surveillance des maladies transmissibles : définitions de cas et méthodes de surveillance particulières à chaque maladie. Rapport hebdomadaire des maladies au Canada 1991;17(S3).

4. Santé Canada. Définitions de cas de maladies faisant l'objet d'une surveillance nationale. RMTC. 2000;26(S3).

5. Agence de la santé publique du Canada. Définitions nosologiques des maladies transmissibles faisant l'objet d'une surveillance nationale. RMTC. 2009;35(S2).

6. Société canadienne de pédiatrie. Programme canadien de surveillance active de l'immunisation. http://www.cps.ca/fr/impact 
7. Statistique de l'état civil - Base de données sur les décès, Statistique Canada, Division de la statistique de la santé, [2000 à 2009]. Consulté le 15 juillet 2013.

8. Statistique de l'état civil du Canada - Estimations provinciales de la population (juillet), Statistique Canada, Division de la statistique de la santé, [estimations intercensitaires définitives de 1924 à 2005; estimations postcensitaires définitives de 2006 à 2009; estimations postcensitaires mises à jour de 2010-2011; estimations postcensitaires provisoires de 2012]. Consulté le 15 juillet 2013.

9. Comité consultatif national de l'immunisation. Prévention de la coqueluche chez les adolescents et les adultes. RMTC. 2003;29(DCC-5).

10. Santé Canada. Conférence de concertation sur la coqueluche. RMTC. 2003;29(S3):1-33.

11. Wiley KE, Zuo Y, Macartney KK, McIntyre PB. Sources of pertussis infection in young infants: a review of key evidence informing targeting of the cocoon strategy. Vaccine. 2013;31(4):618-25.

12. Healy CM, Rench MA, Baker CJ. Importance of timing of maternal combined tetanus, diphtheria, and acellular pertussis (Tdap) immunization and protection of young infants. Clin Infect Dis. 2013;56(4):539-44.

13. Hardy-Fairbanks AJ, Pan SJ, Decker MD, Johnson DR, Greenberg DP, Kirkland KB, et al. Immune responses in infants whose mothers received tdap vaccine during pregnancy. Pediatr Infect Dis J. 2013;32(11):1257-60.

14. Guide canadien d'immunisation [page Web]. Vaccin contre la coqueluche.

http://www.phac-aspc.gc.ca/publicat/cig-gci/p04-pert-coqu-fra.php

15. Klein NP, Bartlett J, Rowhani-Rahbar A, Fireman B, Baxter R. Waning protection after fifth dose of acellular pertussis vaccine in children. N Engl J Med. 2012;367(11):1012-9.

16. Lavine JS, King AA, Bjørnstad ON. Natural immune boosting in pertussis dynamics and the potential for longterm vaccine failure. Proc Natl Acad Sci U S A. 2011;108(17):7259-64.

17. Cherry JD. Epidemic pertussis in 2012 - the resurgence of a vaccine-preventable disease. N Engl J Med. 2012;367(9):785-7.

18. Mooi FR, van der Maas NA, De Melker HE. Pertussis resurgence: waning immunity and pathogen adaptation - two sides of the same coin. Epidemiol Infect. 2013 Feb;13:1-10.

19. Atwell JE, Van Otterloo J, Zipprich J, Winter K, Harriman K, Salmon DA, et al. Nonmedical vaccine exemptions and pertussis in California, 2010. Pediatrics. 2013;132(4):624-30.

20. Laroche J, Gendron MP, Abdel-Motagally M. Impact of validating respondent responses on national immunization coverage estimates [affiche]. Canadian Immunization Conference. 2012 Dec.

\section{Remarques et remerciements}

Les données statistiques de l'état civil ont été fournies à l'Agence par Statistique Canada avec le consentement des registres de statistiques de l'état civil des provinces et des territoires, dont la coopération est grandement appréciée. Bien que des parties du présent rapport soient basées sur des données fournies par l'Institut canadien d'information sur la santé, les analyses, les conclusions, les opinions et les déclarations qui sont exprimées sont celles de l'Agence et pas nécessairement celles de l'Institut. Les estimations provinciales et territoriales présentées par l'Agence peuvent être différentes de celles publiées par les provinces et les territoires en raison des données variables utilisées au moment d'effectuer les analyses. Nous soulignons avec reconnaissance la participation aux activités de déclaration de routine de la coqueluche des fournisseurs de soins de santé, des responsables de la santé publique et des laboratoires du pays. Nous voulons aussi remercier toutes les personnes qui ont collaboré à l'élaboration du présent rapport, notamment : Vicky Springman (Agence), Monique St-Laurent (Agence), John Spika (Agence), Julie Bettinger (programme IMPACT), Scott Halperin (programme IMPACT), Danuta Skowronski (Colombie-Britannique), Monika Naus (Colombie-Britannique), Theresa St. Jean (Alberta), Kimberley Simmonds (Alberta), Helen Bangura (Saskatchewan), Valerie Mann (Saskatchewan), Patricia Caetano (Manitoba), Shelley Deeks (Ontario), Jastej Dhaliwal (Nouveau-Brunswick), Carolyn Sanford (Île-du-Prince-Édouard), Anne Neatby (Île-du-Prince-Édouard), Cathy O'Keefe (Terre-Neuve-et-Labrador), Gillian Butler (Terre-Neuve-et-Labrador), Beverly A. Billard (Nouvelle-Écosse), Lori Strudwick (Yukon), Karolina 
Machalek (Agence/Yukon), Heather Hannah (Territoires du Nord-Ouest), Bryany Denning (Territoires du NordOuest), Carolina Palacios (Nunavut), et Angie Mullen (Nunavut).

\section{Déclaration de conflit d'intérêts}

II n'y a aucun conflit d'intérêts à déclarer.

\section{Financement}

Ce travail a été appuyé par l'Agence de la santé publique du Canada. 